mansasa

glyndîn

Glyndŵr University

Glyndŵr University Research Online

Computing

Computer Science

$1-1-2009$

\title{
Performance Evaluation of The Split Transmission in Multihop Wireless Networks
}

Wanqing Tu

Glyndwr University, w.tu@glyndwr.ac.uk

Vic Grout

GlyndwrUniversity, v.grout@glyndwr.ac.uk

Follow this and additional works at: http://epubs.glyndwr.ac.uk/cair

Part of the Computer Engineering Commons

\section{Recommended Citation}

Tu, W. \& Grout, V., (2009) 'Performance Evaluation of The Split Transmission in Multihop Wireless Networks'.( Proceedings of the 2nd IEEE/ACM/BCS/IET International Workshop on Future Multimedia Networking FMN 2009 on 22-23 June 2009), held in Coimbra, Portugal.

This Article is brought to you for free and open access by the Computer Science at Glyndîr University Research Online. It has been accepted for inclusion in Computing by an authorized administrator of Glyndŵr University Research Online. For more information, please contact d.jepson@glyndwr.ac.uk. 


\title{
Performance Evaluation of The Split Transmission in Multihop Wireless Networks
}

\begin{abstract}
Multimedia applications in multihop wireless networks have great market potential. Multiple channels and multiple radios are commonly used for exploring multimedia transmissions in multihop wireless networks. Split transmission allows multiple channels attached to different radios simultaneously to be used, and so to achieve a fundamentally improved transmission capacity. The goal of this paper is to present a theoretical background to justify the improved performance of split transmission. We believe that this is the first attempt to consider split transmission in theory.
\end{abstract}

\section{Keywords}

wireless multimedia, split transmission, performance evaluation, multiple channels, multiple radios

\section{Disciplines \\ Computer Engineering}

\section{Comments}

This paper was presented at the 2nd IEEE/ACM/BCS/IET International Workshop on Future Multimedia Networking (FMN 2009) on 22-23 June 2009, held in Coimbra, Portugal. It was published by the University of Plymouth and the symposium proceedings will be available at http://www.cscan.org (C) 2009 Springer This version of the work is the authors final draft post-refereeing. The work was published in Volume 5630 of Lecturer Notes in Computer Science pages 72-82 by Springer Berlin/Heidelberg in 2009. The original publication is available at http:/ / www.springerlink.com 


\title{
Performance Evaluation of The Split Transmission in Multihop Wireless Networks
}

\author{
Wanqing Tu and Vic Grout \\ Centre for Applied Internet Research, \\ School of Computing and Communications Technology, \\ Glyndwr University, UK. \\ $\{$ w.tu,v.grout $\} @ g l y n d w r . a c . u k$
}

\begin{abstract}
Multimedia applications in multihop wireless networks have great market potential. Multiple channels and multiple radios are commonly used for exploring multimedia transmissions in multihop wireless networks. Split transmission allows multiple channels attached to different radios simultaneously to be used, and so to achieve a fundamentally improved transmission capacity. The goal of this paper is to present a theoretical background to justify the improved performance of split transmission. We theoretically study and prove that, by using the split transmission, the worst-case delay is decreased to $\frac{\sigma \rho_{k-1}}{L C_{m-1} C_{k-1}}$ of the one without using the split transmission; the average throughput is increased to $\frac{1}{1-\prod_{j=0}^{k-1} \alpha_{j}}$ times of the one without using the split transmission; the average delay jitter is decreased to $\frac{C_{k-1} C_{\rho}}{C_{m-1}\left[C_{\rho}+L(\rho+C)\right]}$ of the one without using the split transmission. We believe that this is the first attempt to consider split transmission in theory.
\end{abstract}

Key Words: Wireless Multimedia, Split Transmission, Performance Evaluation, Multiple Channels, Multiple Radios.

\section{INTRODUCTION}

A multihop wireless network (e.g. an ad hoc network, sensor network, mesh network) is a self-organized and self-configured wireless architecture in which two 
wireless nodes communicate through a number of intermediate nodes, whose functions are to relay data one by one. Multihop wireless networks have multiple advantages: reliable coverage, robustness, and easy maintenance to facilitate interactive multimedia communications that are in great market demand. Examples of multihop wireless multimedia applications are online games, wireless video conferences, online exchange, real-time monitoring of activities at homes and in offices, etc. However, communications over wireless link area subject to channel fading, multipath fading, and interference from background noise and neighbors, which degrades the performance of multimedia communications.

To improve the degraded multimedia performance, a number of research studied multimedia transmission algorithms/schemes in multihop wireless networks. One approach [1-3] focuses on switching multiple channels on the same radio interfaces. An alternative line of research [4-9] exploits the advantage of multiple radio interfaces for multimedia transmissions. However, few of them suits for an interactive multimedia transmission which highly requires for a high quality transmission in a real-time way. We designed a split multimedia transmission in [10]. The algorithm uses multiple radio interfaces in parallel to transmit a multimedia stream that will suffer from bottleneck. Our simulation results in [10] observed that the algorithm fundamentally improves multimedia performance. In this paper, we present a theoretical background to explain the performance improvement of split transmission. We believe this is the first attempt to study the split transmission in theory.

The acceptable performance of interactive multimedia communications requires uninterrupted and distortionless content reception within stringent delays. These requirements can be concluded as three metrics of worst-case delay, average throughput, and average delay jitter. An end-to-end delay is the time taken for a packet to transmit across a network from a source to a destination. It is the summation of the packet's transmission delay, propagation delay and processing delay. A worst-case delay, evaluated in real time, is the longest endto-end delay of a multimedia transmission. Throughput is the average rate of successful message delivery over a channel. Average throughput affects the definition of multimedia playback. Delay jitter is defined as the end-to-end delay difference between two consecutive packets. Average delay jitter evaluates the continuity of a multimedia transmission. Large delay jitter causes interrupted multimedia playback. This paper mathematically evaluates these three metrics in a multihop split multimedia transmission. 
There are two classical ways to model multimedia traffic: a leaky bucket [11$12]$ and a $(\sigma, \rho)$ regulator [13-14]. The leaky bucket enforces a rigid output pattern at the average rate $\rho$ not matter how bursty the input traffic is. For multimedia traffic, a more flexible mechanism is required to process large burstiness that allows the short delay output, preferably one that does not lose data. The $(\sigma, \rho)$ regulator introduces burstiness into the traffic model. Thus, we employ the $(\sigma, \rho)$ regulator to model multimedia traffic in our theoretical evaluation, and present the following results for a multimeida stream $f$ transmitted in a $k$-hop wireless networks. (Suppose there are $m$ radios selected by the split transmission.)

- The worst-case delay of split transmission is upper bounded by $\frac{k \sigma}{C_{m-1}}+\frac{k L}{C_{m-1}}$, where $L$ is the average packet size of the multimedia stream and $C_{m-1}$ is the transmission capacity of the last (i.e. $(m-1)$ th) selected channel; the worst-case delay of non-split transmission is $k L \frac{1+C_{k-1}^{2}}{C_{k-1} \rho_{k-1}}$, where $\rho_{k-1}$ and $C_{k-1}$ are the average transmission rate and the output capacity at the last (i.e. $(k-1)$ th) hop;

- The average throughput of split transmission is $\rho+\frac{\sigma}{\Delta t}$, where $\Delta t$ is the time that has elapsed since the initial transmission of $f$; the average throughput of non-split transmission is upper bounded by $\left(1-\prod_{j=0}^{k-1} \alpha_{j}\right)\left(\frac{\sigma}{\Delta t}+\rho\right)$, where $\alpha_{j}$ is the packet loss rate at the $j$ th hop $(j \in[0, k-1])$;

- The average delay jitter of split transmission is $k \frac{\rho-C_{m-1}}{C_{m-1}}\left[t_{l}-t_{l-1}\right]$, where $t_{l}$ and $t_{l-1}$ are the transmission times of the $l$ th and the $(l-1)$ th packets; the average delay jitter of non-split transmission is $\frac{k}{\rho}\left(\frac{\rho}{C_{k-1}}-1\right)\left[\left(\frac{\rho}{C_{k-1}}-\right.\right.$ 1) $\left.\Delta t_{k-1, p-1}+\left(t_{l}-t_{l-1}\right)\right]$, where $\Delta t_{k-1, p-1}$ is the time that has elapsed to transmit the $(p-1)$ th packet at the $(k-1)$ th hop since $f$ 's initial transmission.

The theoretical evaluation proves that the split transmission is an effective way to achieve short delay, high throughput, and continuous wireless multimedia performance. While the modern technology is still developing to open wider implementation for split transmission, the algorithm promises to be used currently to solve burstiness without changing the existed wireless hardware and MAC protocols.

The following of the paper is organized as follows. Section 2 introduces the related work. Section 3 briefly details the split transmission algorithm. In section 4, we analyze the performance of worst-case delay, average throughput, and average delay jitter. Section 5 concludes this paper. 


\section{Related Work}

Related studies for wireless multimedia transmission mainly focus on algorithm /protocol design to achieve some anticipated performance. These work can be classified as multi-channel single interface schemes and multi-channel multiinterface schemes.

Protocols in [1-3] are multi-channel single interface schemes that make use of the capacities of multiple channels on the same radio interface. [1] proposed SSCH (slotted seeded channel hopping) that slots the time for nodes to hop between multiple channels without incurring transmission interference. A global time is required to synchronize channel selection between nodes. Also hopping between channels creates transmission jitter. J. So et al [2] designed a medium access control protocol that dynamically uses temporal synchronization to solve the hidden terminal problem in ad hoc networks. The protocol also requires a complex global clock. S. Wu et al [3] proposed a RTS/CTS like reservation mechanism to dynamically assign channels to mobile nodes in an "on-demand" way. Generally, multi-channel single interface protocols cannot avoids collision because multiple channels attach to the same interface. Complex algorithms are required to decrease collision and as well to maintain a global clock.

Another approach is to utilize the advantage of multiple interfaces to assign a difference radio interface to each individual channel. P. Kyasanur et al [8] presented a channel assignment protocol in the context of multi-radio wireless mesh networks. Each node has some fixed channel and is also dynamically connected to other channels over short time. A sender then adapts to a receiver by changing its temporary channel to the receiver's fixed channel. A. Adya et al [4] presented a multi-radio unification protocol for multihop wireless mesh networks with the goal to optimize local spectrum utilization through intelligent channel selection. A. Raniwala et al [6] proposed a centralized greedy solution that accesses wireless links in decreasing order of link loads. The solution cannot deal with dynamic traffic load. Hence, A. Raniwala et al [7] extended this study to a distributed algorithm. The designed architecture (called Hyacinth) uses local traffic load information to dynamically assign channels and route packets. In general, through selecting an individual optimal channel for each traffic, the improved performance achieved by most multi-channel multi-interface schemes is limited by each individual channel's capacity.

Our split transmission [10] aggregates capacities from multiple radios for the use of one multimedia stream. The performance is considerably improved as 
compared to other algorithms. We are interested in explaining such improvement theoretically in this paper.

\section{Split Transmission}

The motivation of split transmission is to simultaneously use multiple interferencefree channels to transmit QoS guaranteed multimedia streams. When a wireless node detects a coming overload in an output channel because of transmitting a multimedia stream $f$, it selects $m$ radios that are used to transmit the stream together. Split transmission guarantees that the minimum number of channels with enough cumulative capacity are selected to carry the multimedia stream. That is, if $r$ channels are selected, the following expression exists.

$$
\left\{\begin{array}{l}
C(\hat{0}, t)+C(\hat{1}, t)+\ldots+C(\hat{i}, t)+\ldots+C((r \hat{-} 1), t) \geq r_{f}, \\
C(\hat{0}, t)+C(\hat{1}, t)+\ldots+C(\hat{i}, t)+\ldots+C((r \hat{-} 2), t) \leq r_{f}
\end{array}\right.
$$

where $t$ is the time at which the node uses the split transmission, $r_{f}$ is $f$ 's transmission rate, and $C(\hat{i}, t)$ is the $i$ th selected channel's capacity at the time $t$. Based on each selected channel's individual capacity, the wireless node splits the multimedia stream into $r$ subflows. Each subflow has a transmission rate $r_{i}$ $(i \in[0, r-1])$ matching the capacity of one selected channel $\hat{C}_{i}$. Namely, $r_{i}=\hat{C}_{i}$. Then as illustrated in Fig. 1, these $r$ subflows will be transmitted through the $r$ selected channels in parallel.

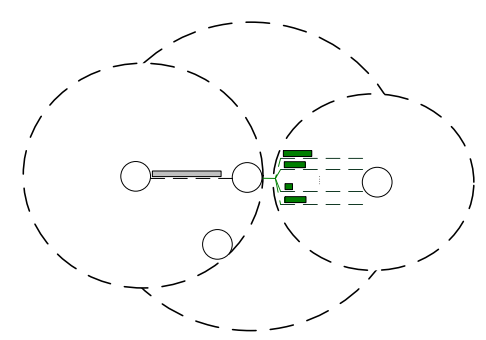

Fig. 1. An example of split transmission.

Split transmission has been proved to be effective in improving wireless multimedia transmission performance by our simulations in [10]. In this paper, we mathematically study the split transmission to present a theoretical background for the improved performance. 


\section{Performance Evaluation for The Split Transmission}

To implement the analysis, as we have introduced, we use a $(\sigma, \rho)$ regulator to model multimedia traffic. In [13], the $(\sigma, \rho)$ regulator is defined as Given $\sigma>0$ and $\rho>0$, for an input flow with the rate function $R$, the following inequality exists if and only if $y \geq x$ for all $x$ and $y$,

$$
\int_{x}^{y} R d t \leq \sigma+\rho(y-x),
$$

where $\rho$ is the flow's average input rate and $\sigma$ is the flow's burst constraint. (1) shows that the upper bound of the amount of multimedia traffic input into the network between the times $y$ and $x$ is decided by the traffic burstiness $\sigma$ and the traffic average transmission rate $\rho$.

Before we analyze the performance of worst-case delay, average throughput, and average delay jitter, we list the symbols that will be used for analysis in Table 1 .

Table 1. Symbol List

\footnotetext{
$f \quad$ Represent a multimedia stream.

$m$ The number of selected channels/interfaces in the split transmission.

$\dot{C}_{i} \quad$ The capacity of the $i$ th selected channel, $i \in[0, m-1]$.

$k$ The number of hops from a sender to a receiver in the multihop wireless network.

$C_{j}$ The output capacity at the $j$ th hop in the non-split transmission, $j \in[0, k-1]$.

$L$ The average packet size of $f$.

$\Delta t$ The elapsed transmission time since $f$ is initially transmitted.

$p \quad$ The total number of packets in $f$.

$\Delta t_{l}$ The elapsed transmission time when transmitting the $l$ th packet, $l \in[0, p-1]$.

$\Delta t_{j, l}$ The elapsed transmission time when transmitting the $l$ th packet of the $j$ th hop.
}

\subsection{The Worst-Case Delay}

The worst-case delay, evaluated in real time, is the longest end-to-end delay between a sender and a receiver. We first consider the worst-case delay in a single hop wireless network. According to the definition of $(\sigma, \rho)$ regulator, the transmission rate of $f$ is $R \sim(\sigma, \rho)$. When $f$ is split into $m$ subflows that are denoted as $f_{i}(i \in[0, m-1])$, each subflow's transmission rate satisfies $R_{i} \sim\left(\sigma_{i}, \rho_{i}\right)$, 
where $R_{i}, \sigma_{i}$, and $\rho_{i}$ are $f_{i}$ 's input rate, burstiness, and average transmission rate respectively. Use $C_{i}$ to represent the $i$ th selected channel's available capacity. The transmission delay of the $i$ th subflow at the time $t$ is

$$
D_{i}=\frac{\left[\sigma_{i}+\rho_{i} \Delta t\right]-\left[C_{i} \Delta t-L\right]}{C_{i}},
$$

where $\Delta t=t-t_{0}\left(t_{0}\right.$ is the time that $f$ is initially transmitted by the node) and $L$ is the average packet size of $f$. The item $\left[\sigma_{i}+\rho_{i} \Delta t\right]$ is the upper bound of $f$ 's input data amount in the period $\Delta t$. The item $\left[C_{i} \Delta t-L\right]$ is the amount of $f$ 's output data in the period $\Delta t$.

Based on (2) and $C_{i} \geq \rho_{i}$, it can be inferred that the worst-case delay of $f_{i}$ is $\ddot{D}_{i} \leq \frac{\sigma_{i}+L}{C_{i}}$. Moreover, the worst-case delay $\ddot{D}_{i}$ appears at the time $\left(t_{0}+\frac{L}{C_{i}}\right)$ and decreases to 0 after a period of $\frac{\sigma_{i}+L}{C_{i}-\rho_{i}}$.

Considering all of the $m$ split subflows, the worst-case delay in a single hop wireless network is $\ddot{D}_{s}=\max \left\{\ddot{D}_{i} \mid i \in[0, m-1]\right\}=\max \left\{\frac{\sigma_{i}+L}{C_{i}} \mid i \in[0, m-1]\right\}$. Without loss of generality, we assume $C_{0} \geq C_{1} \geq C_{2} \geq \ldots \geq C_{m-1}$ for the $m$ subflows. This assumption indicates $\frac{\sigma}{C_{m-1}} \geq \max \left\{\frac{\sigma_{i}}{C_{i}}\right\}$. Hence, we have

$$
\ddot{D}_{s} \leq \frac{\sigma}{C_{m-1}}+\max \left\{\frac{L}{C_{i}}\right\} \leq \frac{\sigma}{C_{m-1}}+\frac{L}{C_{m-1}} .
$$

We now extend our analysis into a $k$-hop $(k>1)$ wireless network. The split transmission enables subflows to be transmitted without queueing. Hence, an end-to-end delay is the summation of packet delays at all hops from a sender to a receiver. That is, the worst-case delay when $f$ experiences $k$-hop split transmission is

$$
\ddot{D} \leq k \ddot{D}_{s} \leq \frac{k \sigma}{C_{m-1}}+\frac{k L}{C_{m-1}} .
$$

We next analyze the worst-case delay of multimedia traffic without employing split transmission. The end-to-end delay in a single hop wireless network is

$$
D_{s}^{\prime}=\frac{(\sigma+\rho \Delta t)-C\left(\Delta t-\frac{L}{\rho}-\frac{L}{\rho-C}\right)}{C},
$$

where $C$ is the available capacity of $f$ 's output channel. In a $k$-hop wireless network, based on the expression above, the packet transmission delay at the $j$ th hop is

$$
D_{j}^{\prime}=\frac{\left(\sigma_{j}+\rho_{j} \Delta t\right)-C_{j}\left(\Delta t-\frac{L}{\rho_{j}}-\frac{L}{\rho_{j}-C_{j}}\right)}{C_{j}},
$$

where $j \in[0, k-1], \sigma_{j}$ and $\rho_{j}$ are $f$ 's burstiness and average transmission rate at the $j$ th hop, and $C_{j}$ is output capacity at the $j$ th hop. It shows that the total end-to-end delay when $f$ transmits $k$ hops is calculated by

$$
D^{\prime}=\sum_{j=0}^{k-1} D_{j}^{\prime}=\sum_{j=0}^{k-1} \frac{\left(\sigma_{j}+\rho_{j} \Delta t\right)-C_{j}\left(\Delta t-\frac{L}{\rho_{j}}-\frac{L}{\rho_{j}-C_{j}}\right)}{C_{j}} .
$$


To develop (6), we know that the $k$-hop non-split transmission has these characteristics: $\sigma_{j} \geq \sigma_{j+1}, \rho_{j} \geq \rho_{j+1}, C_{j} \geq C_{j+1}$, and $C_{j} \Delta t=\sigma_{j+1}+\rho_{j+1} \Delta t$. Hence, it can be inferred that

$$
\begin{aligned}
D^{\prime} & \leq \frac{\sigma_{0}+\rho_{0} \Delta t}{C_{0}}+\ldots+\frac{\sigma_{k-1}+\rho_{k-1} \Delta t}{C_{k-1}}-\left[k \Delta t-k \frac{L}{C_{k-1} \rho_{k-1}}-k \frac{L}{C_{k}\left(\rho_{k}-C_{k}\right)}\right] \\
& \leq k \Delta t-\left[k \Delta t-k \frac{L}{C_{k-1} \rho_{k-1}}-k \frac{L}{C_{k-1}\left(\rho_{k-1}-C_{k-1}\right)}\right] \leq k L \frac{1+C_{k-1}{ }^{2}}{C_{k-1} \rho_{k-1}} .
\end{aligned}
$$

The worst-case delay of non-split multimedia transmission in a $k$-hop wireless network is therefore

$$
\ddot{D}^{\prime}=k L \frac{1+C_{k-1}^{2}}{C_{k-1} \rho_{k-1}} .
$$

To calculate the worst-case delay improvement of split transmission, we have

$$
\frac{\ddot{D}}{\ddot{D}^{\prime}}=\frac{(\sigma+L) C_{k-1} \rho_{k-1}}{C_{m-1} L\left(1+C_{k-1}^{2}\right)} \approx \frac{\sigma \rho_{k-1}}{L C_{m-1} C_{k-1}} .
$$

Since $L>>m$, we have $\sigma<L C_{m-1}$. Usually, $\rho_{k-1} \leq C_{k-1}$. Hence, $\frac{\ddot{D}}{\ddot{D}^{\prime}}<1$. Furthermore, $\ddot{D}$ comes into existence at the time $t_{0}+\frac{L}{C_{m-1}}$. With the transmission continues, the end-to-end delay of split transmission reduces to 0 while the end-to-end delay of non-split transmission increases more and more to the maximum value of $k L \frac{1+\left(C_{k-1}\right)^{2}}{C_{k-1} \rho_{k-1}}$.

\subsection{The Average Throughput}

The average throughput evaluates the playback quality (i.e. video definition and audio articulation) of multimedia traffic. We first consider the average throughput of split transmission in a single hop wireless network. Each of the $m$ selected channels has enough capacity to output one split subflow. It shows that the total output packets of $f$ through the $m$ channels should be equal to $f$ 's input amount. Thus, in a single hop wireless network,

$$
\sum_{i=0}^{m-1}\left[C_{i}\left(\Delta t-\frac{L}{\rho_{i}}\right)\right]=\sigma+\rho \Delta t
$$

Based on the above equation and $\rho_{i} \leq \rho$, we have

$$
\sum_{i=0}^{m-1} C_{i}=\frac{(\sigma+\rho \Delta t) \rho}{\rho \Delta t-L} \approx \rho+\frac{\sigma}{\Delta t}
$$

For the average throughput in a $k$-hop wireless network, multimedia transmission at each hop is able to output all received packets. It means that each hop achieves the average throughput in (9). Hence, the average through of the split transmission after $k$ hops is $\bar{T}=\rho+\frac{\sigma}{\Delta t}$. 
We now consider the average throughput of non-split transmission in a $k$ hop wireless network. Assume the loss rate at the $j$ th hop transmission is $\alpha_{j}$ $(j \in[0, k-1])$. The following equation is established based on the fact that the difference between the input amount and the output amount is equal to the amount of lost packets.

$$
\sigma+\rho \Delta t-C_{k-1}\left(\Delta t-\frac{L}{\rho}-\frac{L}{\rho-C_{k-1}}\right)=\prod_{j=0}^{k-1} \alpha_{j}(\sigma+\rho \Delta t) .
$$

This equation infers that the average throughput with the split transmission is

$$
T^{\prime} \leq \frac{\rho(\sigma+\rho \Delta t)\left(1-\prod_{j=0}^{k-1} \alpha_{j}\right)}{\rho \Delta t-2 L} \approx\left(1-\prod_{j=0}^{k-1} \alpha_{j}\right)\left(\frac{\sigma}{\Delta t}+\rho\right) .
$$

To compare the average throughput of split transmission and non-split transmission in a $k$-hop wireless network, we use the following equations.

$$
\frac{T}{T^{\prime}}=\frac{1}{1-\prod_{j=0}^{k-1} \alpha_{j}} .
$$

As compared to non-split transmission, the improvement in the average throughput of split transmission increases when $f$ traverses more numbers of hops. It proves that the spit transmission suits to multihop wireless multimedia transmissions.

\subsection{The Average Delay Jitter}

The average delay jitter is the metric that evaluates the continuity of multimedia traffic. Small average delay jitter benefits smoothing video playback and uninterrupted audio reception. According to (2), the end-to-end delay of split multimedia transmission decreases to 0 after a period of $\tau=\max \left\{\frac{\sigma_{i}+L}{C_{i}-\rho_{i}} \mid i \in[0, m-1]\right\}$. The average delay jitter is therefore 0 after the period $\tau$. Hence, we focus on analyzing the average delay before the time $\left(t_{0}+\tau\right)$.

Suppose there are totally $p_{i}\left(p_{i} \in N\right)$ packets transmitted from the sender to the receiver through the $i$ th selected channel. We use $D_{i, l}$ and $D_{i,(l-1)}(l \in$ $\left.\left[0, p_{i}-1\right]\right)$ to represent the end-to-end delays of the $l$ th and the $(l-1)$ th packets transmitted through the $i$ th channel. Then, the delay jitter between the $j$ th and the $(j-1)$ th packets is $J_{i, l}=D_{i, l}-D_{i,(l-1)}$. According to (2),

$$
J_{i, l}=D_{i, l}-D_{i,(l-1)}=\left(\frac{\rho_{i}}{C_{i}}-1\right)\left(\Delta t_{l}-\Delta t_{l-1}\right) .
$$

In the split multimedia transmission, packets can be transmitted once they arrive without queueing. Hence, $\Delta t_{l}-\Delta t_{l-1}=t_{l}-t_{l-1}$. It infers that $J_{i, l}=\left(\frac{\rho_{i}}{C_{i}}-\right.$ 
1) $\left(t_{l}-t_{l-1}\right)$. Thus, the average delay jitter of split multimedia transmission in a single hop wireless network is

$$
\begin{gathered}
\bar{J}_{s}=\frac{\sum_{i=0}^{m-1} \sum_{l=0}^{p_{i}-1} J_{i, l}}{\sum_{i=0}^{m-1} p_{i}}=\frac{\sum_{i=0}^{m-1} \sum_{l=0}^{p_{i}-1}\left(\frac{\rho_{i}-C_{i}}{C_{i}}\right)\left(t_{l}-t_{l-1}\right)}{\sum_{i=0}^{m-1} p_{i}} \\
\leq \frac{\sum_{i=0}^{m-1} \sum_{l=0}^{p_{i}-1}\left(\frac{\rho-C_{m-1}}{C_{m-1}}\right)\left(t_{l}-t_{l-1}\right)}{\sum_{i=0}^{m-1} p_{i}} 1=\frac{\rho-C_{m-1}}{C_{m-1}}\left(t_{l}-t_{l-1}\right) .
\end{gathered}
$$

Based on (12), the average delay jitter when $f$ is in a $k$-hop wireless split transmission is

$$
\bar{J}=\sum_{i=0}^{k-1} J_{i}=k J_{i}=k \frac{\rho-C_{m-1}}{C_{m-1}}\left[t_{l}-t_{l-1}\right]
$$

We now consider the average delay jitter of non-split multimedia transmission. Suppose $p$ is the total amount of packets that $f$ has. Obviously, $p=$ $\sum_{i=0}^{m-1} p_{i}$. According to our analysis in (5), the delay jitter $J_{j, l}^{\prime}$ of the $l$ th packet at the $j$ th hop is

$$
J_{j, l}^{\prime}=\frac{\rho_{j}-C_{j}}{C_{j}}\left(\Delta t_{l}-\Delta t_{l-1}\right) .
$$

For the non-split transmission, $\Delta t_{j, l}-\Delta t_{j,(l-1)}=\frac{\left(\rho_{j}-C_{j}\right) \Delta t_{j, l}}{C_{j}}+t_{l}-t_{l-1}$, where $\frac{\left(\rho_{j}-C_{j}\right) \Delta t_{j, l}}{C_{j}}$ is the queueing delay of the packets that are transmitted at the time $t_{j, l}$. Hence, $J_{j, l}=\frac{\rho_{j}-C_{j}}{C_{j}}\left[\frac{\rho_{j}-C_{j}}{C_{j}} \Delta t_{j, l}+t_{l}-t_{l-1}\right]$. It shows that the delay jitter of the $l$ th packet after a $k$-hop transmission is

$$
\begin{aligned}
& J_{l}^{\prime}=\sum_{j=0}^{k-1} J_{j, l}=\left[\left(\frac{\rho_{0}}{C_{0}}-1\right)^{2}+\left(\frac{\rho_{1}}{C_{1}}-1\right)^{2}+\ldots+\left(\frac{\rho_{k-1}}{C_{k-1}}-1\right)^{2}\right] \Delta t_{k-1, l}+\left[\left(\frac{\rho_{0}}{C_{0}}-1\right)+\left(\frac{\rho_{1}}{C_{1}}-1\right)\right. \\
& \left.\quad+\ldots+\left(\frac{\rho_{k-1}}{C_{k-1}}-1\right)\right]\left(t_{l}-t_{l-1}\right) \leq k\left(\frac{\rho}{C_{k-1}}-1\right)^{2} \Delta t_{j, l}+k\left(\frac{\rho}{C_{k-1}}-1\right)\left(t_{l}-t_{l-1}\right)^{2}
\end{aligned}
$$

Therefore, the average delay jitter of the flow $f$ in the $k$-hop non-split transmission is

$$
\bar{J}^{\prime}=\frac{\sum_{l=0}^{p-1} J_{l}^{\prime}}{p} \leq \frac{k}{p}\left[\left(\frac{p}{C_{k-1}}-1\right)^{2} \Delta t_{k-1, p-1}+\left(\frac{\rho}{C_{k-1}}-1\right)\left(t_{l}-t_{l-1}\right)\right] .^{3}
$$

To compare these two average delay jitter, we have

$$
\begin{gathered}
\frac{J^{\prime}}{J}=\frac{C_{m-1}\left(\frac{\rho}{C_{k-1}}-1\right)}{k\left(\rho-C_{m-1}\right)\left[t_{l}-t_{l-1}\right]}\left[\frac{k\left(\frac{\rho}{C_{k-1}}-1\right) \Delta t_{k-1, p-1}}{p}+\left(t_{l}-t_{l-1}\right)\right] \\
\approx \frac{C_{m-1}\left(\frac{\rho}{C_{k-1}}-1\right)}{k\left(\rho-C_{m-1}\right)\left(t_{l}-t_{l-1}\right)}\left[\frac{\Delta_{k-1, p-1}}{p}+\left(t_{l}-t_{l-1}\right)\right]^{4}
\end{gathered}
$$

\footnotetext{
${ }^{1}$ This inequation is inferred from $\rho_{i} \leq \rho_{0}=\rho$ and $C_{i} \geq C_{m-1}$.

${ }^{2}$ This inequation i inferred from $\rho_{j} \leq \rho_{0} \leq \rho, C_{j} \geq C_{k-1}$, and $\Delta t_{j, l} \leq \Delta t_{k-1, l}$.

${ }^{3}$ It is because $\Delta t_{k-1, l} \leq \Delta t_{k-1, p-1}$

${ }^{4}$ It is because $\rho \geq k\left(\frac{\rho}{C_{k-1}}-1\right)$.
} 


$$
\approx \frac{C_{m-1}\left(\frac{\rho}{C_{k-1}}-1\right)}{p k\left(\rho-C_{m-1}\right)}\left[\Delta t_{k-1, p-1}+1\right]^{5}=\frac{\frac{\rho}{C_{k-1}}-1}{\frac{\rho}{C_{m-1}}-1} \frac{\Delta t_{k-1, p-1}}{k p} .
$$

In order to develop the above expression further, it is inferred that

$\Delta t_{k-1, p-1}=\sum_{j=0}^{k-1}\left[\frac{p L}{C_{j}}+\frac{p L}{\rho_{j}-C_{j}}\right] \geq p L \sum_{j=0}^{k-1}\left[\frac{1}{C_{j}}+\frac{1}{\rho_{j}}\right] \geq p L \sum_{j=0}^{k-1}\left[\frac{1}{C+\frac{1}{\rho}}\right]=\frac{k p L(\rho+C)}{C \rho}$.

Input the above result into the expression of $\frac{J}{J^{\prime}}$, we have

$$
\frac{J}{J^{\prime}} \leq \frac{\frac{\rho}{C_{m-1}}-1}{\frac{\rho}{C_{k-1}}-1} \frac{C_{\rho}}{C_{\rho}+L(\rho+C)} \leq \frac{C_{k-1}}{C_{m-1}} \frac{C_{\rho}}{C_{\rho}+L(\rho+C)} .
$$

\section{Conclusion}

In this paper, we theoretically studied the split transmission through the metrics of worst-case delay, average throughput, and average delay jitter in theory. We found that, through using the split transmission, the worst-case delay is decreased to $\frac{\sigma \rho_{k-1}}{L C_{m-1} C_{k-1}}$ of the one without using the split transmission; the average throughput is increased to $\frac{1}{1-\prod_{j=0}^{k-1} \alpha_{j}}$ times of the one without using the split transmission; the average delay jitter is $\frac{C_{k-1} C_{\rho}}{C_{m-1}\left[C_{\rho}+L(\rho+C)\right]}$ of the one without using the split transmission. It shows that the split transmission achieves short delay, high throughput, and continuous wireless multimedia performance.

The split transmission has no requirement for underlying network architecture and can be easily developed on top of current wireless hardware and MAC protocols. But it needs multiple radio interfaces at one wireless node and the performance improves more if more numbers of radio interfaces are available. While the modern technology enables more than one radio interface at each node and is still working on providing more numbers of radio interfaces, the split transmission occupies multiple radio interfaces only when network situations become bad. A number of overloaded channels in wireless multimedia communications is caused by short-term burstiness due to the variable rate transmission. Hence, the split transmission is very promising to be used currently to achieve high performance wireless multimedia transmission.

\section{References}

1. P. Bahl, R. Chandra, and J. Dunagan, SSCH: Slotted Seeded Channel Hopping For Capacity Improvement In IEEE 802.11 Ad-Hoc Wireless Networks, in Proc. of MOBICOM 2004.

\footnotetext{
${ }^{5}$ It is because $\Delta t_{k-1, p-1} \geq t_{l}-t_{l-1}$.
} 
2. J. So, and N. H. Vaidya, Multi-Channel MAC For Ad-Hoc Networks: Handling Multi-Channel Hidden Terminals Using A Single Transceiver, in Proc. of MOBIHOC 2004.

3. S. Wu, C. Lin, Y. Tseng, and J. Sheu, A New Multi-Channel MAC Protocol With On-Demand Channel Assignment For Multi-Hop Mobile Ad-Hoc Networks, in Proc. of ISPAN 2000.

4. A. Adya, P. Bahl, J. Padhye, A. Wolman, and L. Zhou, A Multi-Radio Unification Protocol for IEEE 802.11 Wireless Networks, in Proc. of Broadnets 2004.

5. M. K. Marina, and S. Das, A Topology Control Approach to Channel Assignment In Multi-Radio Wireless Mesh Networks, in Proc. of Broadnets 2005.

6. A. Raniwala, K. Gopalan, and T. Chiueh, Centralized Channel Assignment And Routing Algorithms For Multi-Channel Wireless Mesh Networks, in Proc. of ACM SIGMOBILE MC2R, 8(2):50C65, 2004.

7. R. Raniwala, and T. Chiueh, Architecture And Algorithms For An IEEE 802.11 Based Multi-Channel Wireless Mesh Network, in Proc. of INFOCOM 2005.

8. P. Kyasanur, and N. Vaidya, Routing and Interface Assignment in Multi-Channel Multi-Interface Wireless Networks, in Proc. of WCNC 2005, 2005.

9. M. Shin, S. Lee, and Y. Kim, Distributed Channel Assignment for Multi-Radio Wireless Networks, in Proc. of IEEE MASS, Vancouver Canada, October 2006.

10. W. Tu, and C. Sreenan, Adaptive Split Transmission for Video Streams in Wireless Mesh Networks, in Proc. of The 2008 IEEE Wireless Communications and Networking Conference (WCNC'08), April 2008, Las Vegas, US.

11. M. Sidi, W. Z. Liu, I. Cidon, and I. Gopal. Congestion Control through Input Rate Regulation. In Proc. of IEEE Globecom 1989, page 1764-1768, November 27-30, 1989.

12. A. E. Echberg, D. T. Luan, and D. M. Lucantoni. An Approach to Controlling Congestion in ATM Networks. International Journal of Digital Analog Communication System, vol. 3, page 199-209, 1990.

13. R. Cruz. A Calculus for Network Delay, Part I: Network Elements in Isolation. IEEE Transaction on Information Theory, vol. 37, no. 1, page 114-131, January 1991.

14. R. Cruz. A Calculus for Network Delay, Part II: Network Analysis. IEEE Transaction on Information Theory, vol. 37, no. 1, page 131-141, January 1991. 